\title{
Network Pharmacology-Based Strategy and Molecular Docking to Explore the Potential Mechanism of Jintiange Capsule for Treating Osteoporosis
}

\author{
Zhao Yang $\mathbb{D}^{\mathbb{B}}$, Zhen-Zhen Yuan $(\mathbb{D}$, and Xin-Long Ma \\ Department of Orthopedics, Tianjin Hospital, Tianjin 300211, China \\ Correspondence should be addressed to Zhao Yang; anny.allan@126.com and Xin-Long Ma; maxinlong86@126.com
}

Received 6 July 2021; Revised 11 November 2021; Accepted 12 November 2021; Published 3 December 2021

Academic Editor: Saheed Sabiu

Copyright (c) 2021 Zhao Yang et al. This is an open access article distributed under the Creative Commons Attribution License, which permits unrestricted use, distribution, and reproduction in any medium, provided the original work is properly cited.

\begin{abstract}
Background. With the advent of ageing population, osteoporosis (OP) has already become a global challenge. Jintiange capsule is extensively applied to treat OP in China. Although recent studies demonstrate that it generates significant effects on strengthening bone, the exact mechanism of the jintiange capsule for treating OP remains unknown. Purpose. To understand the main ingredients of the jintiange capsule, predict the possible targets and the relevant signal transduction pathways, and explore the mechanism of the jintiange capsule for the treatment of OP. Methods. Main ingredients of the jintiange capsule, drug targets, and potential disease targets for OP were obtained from public databases. Molecular biological processes and signaling pathways were determined via bioinformatic analysis, containing protein-protein interaction (PPI), Gene Ontology (GO), and Kyoto Encyclopedia of Genes and Genomes (KEGG). Subsequently, the disease-drug-ingredient-targets-pathways networks were constructed using Cytoscape. According to CytoNCA, core targets were acquired. Finally, the present study conducted molecular docking for better testing the abovementioned results. Results. In the current work, we found that 4 main ingredients of the jintiange capsule, 33 drug targets, 4745 potential disease targets for OP, and 12 overlapping targets were identified. PPI network containing 12 nodes and 25 edges proved that there existed a complex relationship. As revealed by GO functional annotation, the intersected targets were mostly associated with BP, CC, and MF. The targets were enriched to 368 items in BP, 27 items in CC, and 42 items in MF. They mainly included calcium ion homeostasis, calcium channel complex, and calcium channel regulator activity. According to KEGG pathway analysis, the intersected targets were mostly associated with Rap 1, cGMP-PKG, Ras, cAMP, calcium pathways, and so on. Based on the analysis with CytoNCA, we acquired 4 core targets, respectively-CALR, SPARC, CALM1, and CALM2. Besides, 2 core targets, CALR and CALM1, were selected for molecular docking experiments. Molecular docking revealed that the main ingredient, calcium phosphate, had good binding with the CALR protein and CALM1 protein. Conclusion. To conclude, the main ingredient of the jintiange capsule, particularly calcium phosphate, may interact with 2 targets, CALR and CALM1, and regulate multiple signaling pathways to treat $\mathrm{OP}$. Additionally, this also benefits us in further understanding the mechanism of the jintiange capsule for treating OP.
\end{abstract}

\section{Introduction}

Osteoporosis (OP) refers to the systemic progressive skeletal disorder, which is featured by bone tissue microarchitectural deterioration and decreased bone density, causing fracture tendency and bone fragility [1]. The USA has witnessed over 10 million OP cases. Meanwhile, there are 200 million male and female OP cases globally. With the advent of an aging population, OP becomes the worldwide concern influencing the life quality of OP individuals [2]. Therefore, timely diagnosis and treatment of this disease are of great necessity [3].

As the valuable treasure in China, Traditional Chinese Medicine (TCM) exhibits particular effects on preventing and treating diseases [4]. Jintiange capsule, a TCM formula, is extensively applied for the treatment of OP. It contains artificial tiger bone powders prepared from diverse animal bones, whose components and therapeutic efficacy are close 
to those of tiger bone. However, the application of tiger bone is forbidden since tiger is among the list of protected animals in China [5]. Although recent studies also reveal that it exerts significant effects on strengthening bone, the exact mechanism of the jintiange capsule for treating OP remains unknown.

Network pharmacology (NP) is a new discipline based on the theory of system biology, which explores the network of the biological system and selects specific signal nodes to design multitarget drug molecules. First proposed by Hopkins in 2007, NP is a promising approach that can combine information science with systems medicine [6]. NP provides an efficient approach for investigating the synergy of TCM components and their related mechanisms [7]. Apart from that, molecular docking (MD) is a method of drug design through the characteristics of receptors as well as the interaction between receptors and drug molecules. It can be applied in computer-assisted drug discovery, particularly in new drug discovery to treat disorders [8]. The abovementioned two methods provide the effective way to investigate the active sites in drugs and exert important functions in TCM [9]. The present work adopted NP and $\mathrm{MD}$ for comprehending the major components in the jintiange capsule, predicting the possible targets and pathways as well as exploring those mechanisms of the jintiange capsule for treating OP.

\section{Methods}

2.1. Screening for Main Ingredients. The Traditional Chinese Medicine Integrated Database (TCMID, http://www. megabionet.org/tcmid/) was employed to obtain the main ingredients of tiger bone. TCMID is a TCM database with 842 disorders and novel connections, 18203 herbal components, 85 targets, 1356 drugs, and 15 prescriptions [10]. The present study utilized the keyword tiger bone to search the TCMID database. In addition, electronic databases PubMed, Google Scholar, and CNKI were adopted to retrieve relevant studies.

2.2. Screening for Drug Targets. DrugBank database (https:// go.drugbank.com/) was adopted to retrieve drug targets for main ingredients. Those selected ingredients were uploaded into DrugBank database, and targets were collected.

2.3. Potential Disease Targets for OP. OP-associated targets were searched in three human gene databases, including Online Mendelian Inheritance in Man (OMIM, https://www. omim.org/), GeneCards (https://www.genecards.org/), and DisGeNET (https://www.disgenet.org/). Then, we combined all targets obtained in the three databases.

2.4. Acquisition of Overlapping Targets. The targets of tiger bone's main ingredient and potential disease targets for OP obtained from the above 3 databases were applied to obtain the overlapping targets.
2.5. Protein-Protein Interaction (PPI) Network Construction. STRING database (https://string-db.org/), a database that predicted known protein interactions, was utilized for constructing and visualizing the PPI network of the overlapping targets. At the same time, the custom value of STRING was set to 0.2 .

2.6. Gene Ontology (GO) and Kyoto Encyclopedia of Genes and Genomes (KEGG) Pathway Enrichment Analysis. GO and KEGG pathway enrichment analysis were conducted for predicting targets of the jintiange capsule for treating OP by a bioconductor, a data package in $\mathrm{R}$ software (version 4.1.0). $q$ value $\leq 0.05$ was set. Besides, the top $10 \mathrm{GO}$ enrichments and KEGG pathways with higher counts were investigated.

2.7. Construction of Disease-Drug-Ingredient-Targets-Pathways (D-D-I-T-P) Networks. D-D-I-T-P networks were established to comprehend the mechanism of the jintiange capsule for treating OP. The networks were constructed and visualized by Cytoscape 3.8.2 software. CytoNCA, a Cytoscape plugin, was used for performing topological analysis of the PPI network. Thus, the core targets can be obtained.

2.8. Molecular Docking. This study obtained 2D structures for major ingredients in PubChem database (https:// pubchem.ncbi.nlm.nih.gov/). Thereafter, we converted the 2D structures to the MOL2 format by adopting Chem3D software and employed them as the ligands. Additionally, we obtained 3D structures for receptor proteins in RCSB PDB database (https://www.rcsb.org/). Subsequently, the SYBYL$\mathrm{X}$ software was used to achieve MD between the receptors and ligands.

\section{Results}

3.1. Main Ingredients of Tiger Bone. From the TCMID database and literature, a total of 4 ingredients of tiger bone were obtained including calcium phosphate, magnesium phosphate, osseocolla, and fat, respectively.

3.2. Drug Targets of Main Ingredients. Totally, 33 drug targets were retrieved from DrugBank database. The targets of main ingredients are illustrated in Table 1.

3.3. Potential Disease Targets for OP. Based on GeneCards database, a total of 4449 potential disease targets which are known for OP were obtained. Altogether, 1098 potential disease targets were collected form DisGeNET database. Afterwards, 12 potential targets were acquired from OMIM database. After merging, we acquired 4745 potential disease targets.

3.4. The Acquisition of Overlapping Genes. Drug targets were intersected with the disease targets to obtain 12 overlapping targets, suggesting that such targets might exert critical effects on treating OP. As shown in Figure 1, these 
TABLE 1: Targets of tiger bone's main ingredients.

\begin{tabular}{|c|c|c|c|}
\hline Main ingredients & Uniprot ID & Protein & Gene symbol \\
\hline \multirow{33}{*}{ Calcium phosphate } & $\mathrm{P} 41180$ & Extracellular calcium-sensing receptor & CASR \\
\hline & P27797 & Calreticulin & CALR \\
\hline & Q99828 & Calcium and integrin-binding protein 1 & CIB1 \\
\hline & O75340 & Programmed cell death protein 6 & PDCD6 \\
\hline & P30626 & Sorcin & SRI \\
\hline & Q99653 & Calcineurin B homologous protein 1 & CHP1 \\
\hline & P09486 & SPARC & SPARC \\
\hline & P27824 & Calnexin & CANX \\
\hline & P35556 & Fibrillin-2 & FBN2 \\
\hline & P04271 & Protein S100-B & S100B \\
\hline & O14958 & Calsequestrin-2 & CASQ2 \\
\hline & Q15493 & Regucalcin & RGN \\
\hline & Q9UBV8 & Peflin & PEF1 \\
\hline & P06703 & Protein S100-A6 & S100A6 \\
\hline & P13693 & Translationally controlled tumor protein & TPT1 \\
\hline & O75838 & Calcium and integrin-binding family member 2 & CIB2 \\
\hline & Q99584 & Protein S100-A13 & S100A13 \\
\hline & P31415 & Calsequestrin-1 & CASQ1 \\
\hline & Q02818 & Nucleobindin-1 & NUCB1 \\
\hline & P80303 & Nucleobindin-2 & NUCB2 \\
\hline & P0DP23 & Calmodulin & CALM1 \\
\hline & Q75N90 & Fibrillin-3 & FBN3 \\
\hline & P28676 & Grancalcin & GCA \\
\hline & Q9ULU8 & Calcium-dependent secretion activator 1 & CADPS \\
\hline & P0DP24 & Calmodulin-2 & CALM2 \\
\hline & P0DP25 & Calmodulin-3 & CALM3 \\
\hline & Q96FQ6 & Protein S100-A16 & S100A16 \\
\hline & P22676 & Calretinin & CALB2 \\
\hline & Q13938 & Calcyphosin & CAPS \\
\hline & Q86UW7 & Calcium-dependent secretion activator 2 & CADPS2 \\
\hline & Q96L12 & Calreticulin-3 & CALR3 \\
\hline & Q9ULB1 & Neurexin-1 & NRXN1 \\
\hline & P62166 & Neuronal calcium sensor 1 & NCS1 \\
\hline Magnesium phosphate & No ID & No protein & No target \\
\hline Osseocolla & No ID & No protein & No target \\
\hline Fat & No ID & No protein & No target \\
\hline
\end{tabular}

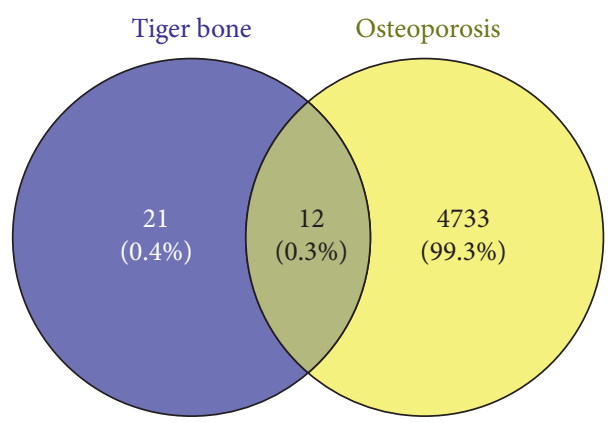

Figure 1: The 12 overlapping targets between the drug and the disease.

overlapping targets include CASR, CALR, SPARC, FBN2, RGN, S100A6, TPT1, NUCB2, CALM1, FBN3, CALM2, and NRXN1.

3.5. PPI Network Construction. We imported 12 overlapping targets between the drug and the disease to STRING database in order to construct the PPI network (Figure 2), containing 12 nodes and 25 edges. Based on the PPI network analysis, such targets had complicated relations.

3.6. GO and KEGG Pathway Enrichment Analysis. To further understand the abovementioned overlapping targets, we utilized the $\mathrm{R}$ software Bioconductor package for GO functional annotation and KEGG pathway analysis. GO terms can 


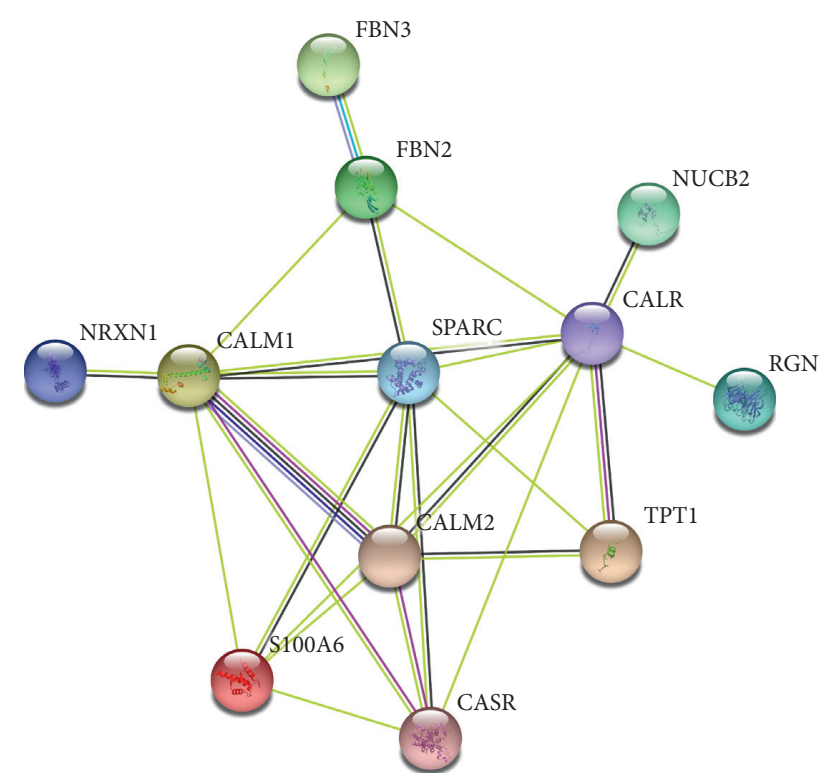

Figure 2: A PPI network containing 12 nodes and 25 edges.

be classified as the following 3 diverse categories: biological process (BP), cellular component (CC), and molecular function (MF). We selected the top 10 according to the $q$ value from small to large, which can be observed in Figure 3. With a total of 368 items in BP, the targets were related to calcium ion homeostasis, divalent inorganic cation homeostasis, response to metalion, and others. The targets were enriched to 27 items in CC, which mainly included collagen-containing extracellular matrix, nuclear envelope, spindle, and calcium channel complex. Concerning 42 items in $\mathrm{MF}$, it mainly contained calcium channel regulator activity, ion channel binding, cytokine activity, phosphatase activator activity, etc.

A total of 45 pathways (Figure 4) were screened out via the KEGG enrichment pathway analysis including Rap1, cGMP-PKG, Ras, cAMP, and calcium pathways. There were several proteins in one pathway. At the same time, one single protein also participated in several pathways. Through the above pathways, such a regulating mechanism revealed the feasibility of using certain major tiger bone ingredients in the treatment of certain disorders, such as OP.

3.7. Construction of D-D-I-T-P Networks. A total of 4 main ingredients were gained from tiger bone. Among 4 ingredients, 3 ingredients could not successfully predict targets. As a result, only 1 ingredient was retained. Furthermore, we chose 1 ingredient, 33 drug targets, and 10 pathways involving most targets for the construction of the D-D-I-T-P networks (Figure 5) by adopting Cytoscape software. According to the analysis with CytoNCA, 4 core targets (Figure 6) were obtained. More details of these core targets are illustrated in Table 2.

3.8. Results of MD. In our study, CALR, SPARC, CALM1, and CALM2 presented a higher degree. Compared with KEGG enrichment analysis results and UniProt database, we chose 2 target genes, CALR and CALM1, to perform
MD experiments. The red part represents the receptor protein. The blue part denotes calcium phosphate. The result indicated that the ingredient, calcium phosphate, enters and binds to active pockets in CALM1 and CALR proteins (Figures 7(a) and 7(c)). The total score was 6.9181 and 5.3811, respectively (Table 3 ). The yellow dotted line represents the connecting hydrogen bond. Calcium phosphate formed 3 hydrogen bonds with A/ARG226.HE and A/ARG226.HH21 of CALR protein (Figure $7(\mathrm{~b})$ ). Simultaneously, calcium phosphate also formed 3 hydrogen bonds with A/ALA73.H and A/ARG74.H of CALM1 protein (Figure $7(\mathrm{~d})$ ). These made the ligand (calcium phosphate) and receptors (CALR protein and CALM1 protein) become a stable complex.

\section{Discussion}

Although several western medicines, such as bisphosphonates, calcitonin, and estrogen, generate a good effect on $\mathrm{OP}$, the important role of TCM could not be ignored. It is wellknown that TCM has been utilizing in China for over 2500 years [11]. Jintiange capsule, the biomimetic medicine prepared from the artificial tiger bone powders, has been recommended to be the efficient therapy by the treatment guidelines for primary OP and OP-related fracture in China in 2017. We acquired 4 main ingredients of tiger bone including calcium phosphate, magnesium phosphate, osseocolla, and fat from the TCMID database. Among the obtained 4 ingredients, only calcium phosphate has 33 targets retrieved from DrugBank database, and the other ingredients have not discovered the relevant targets. Previous study demonstrated that low phosphate or calcium level or uptake could stimulate vitamin D (cholecalciferol) to undergo position 1 secondary hydroxylation within the kidney. As a result, 1,25-dihydroxyvitamin D (calcitriol), the active metabolite, was formed [12]. It was found in the systemic VDR knockdown model that, the phosphate and calcium-rich diet supplemented with lactose prevented osteomalacia and rickets, thus enhancing calcium uptake by the intestine [13]. The above results suggest the necessity of best extracellular phosphate and calcium contents in order to achieve bone and cartilage mineralization [14].

Then, we predicted disease targets from GeneCards, DisGeNET, and OMIM databases, finally obtaining 4745 disease targets. The drug targets in tiger bone were intersected with disease targets, and 12 overlapping targets were obtained, including CASR, CALR, SPARC, FBN2, RGN, S100A6, TPT1, NUCB2, CALM1, FBN3, CALM2, and NRXN1. Then, we constructed a PPI network of overlapping targets and screened 12 nodes and 25 edges via STRING database, revealing that there existed a complex relationship between these targets.

Subsequently, GO and KEGG pathway analysis were conducted on the overlapping targets. As a result, these overlapping targets were enriched in BP, CC, and MF. According to the results, the jintiange capsule treats OP through many BP, mainly including calcium ion homeostasis, divalent inorganic cation homeostasis, response to metal ion, and others. CC mainly included collagen- 


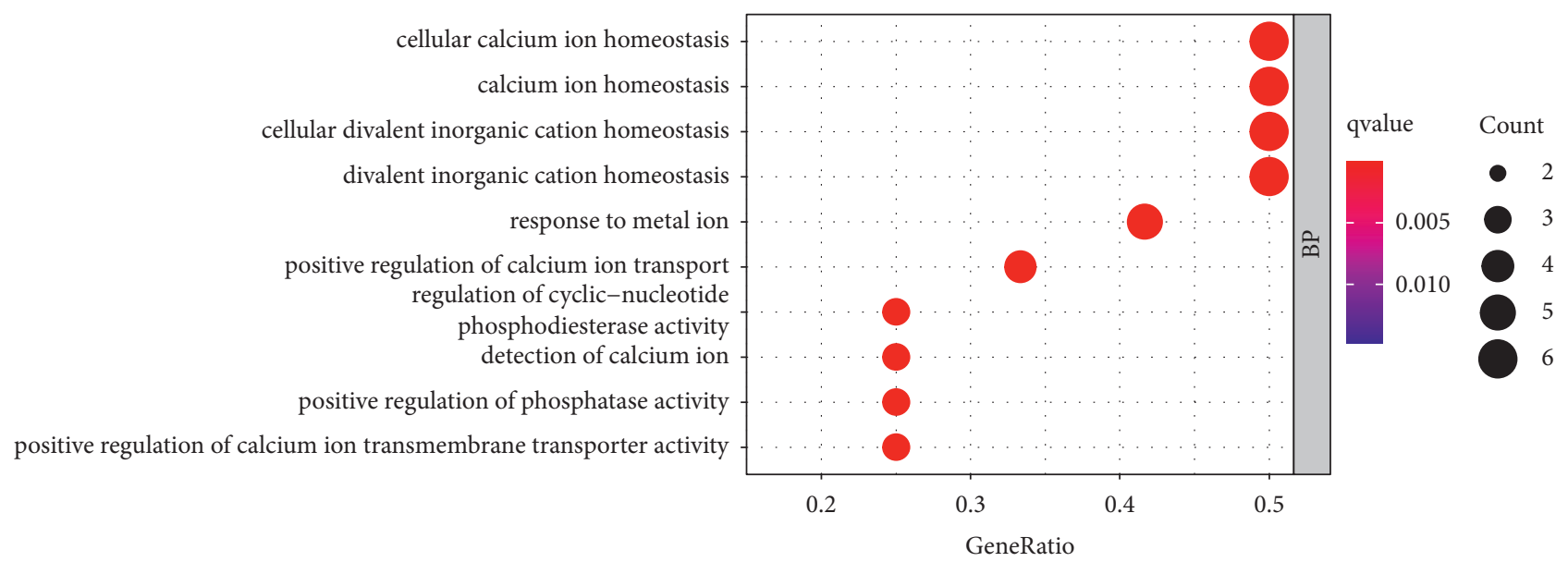

(a)

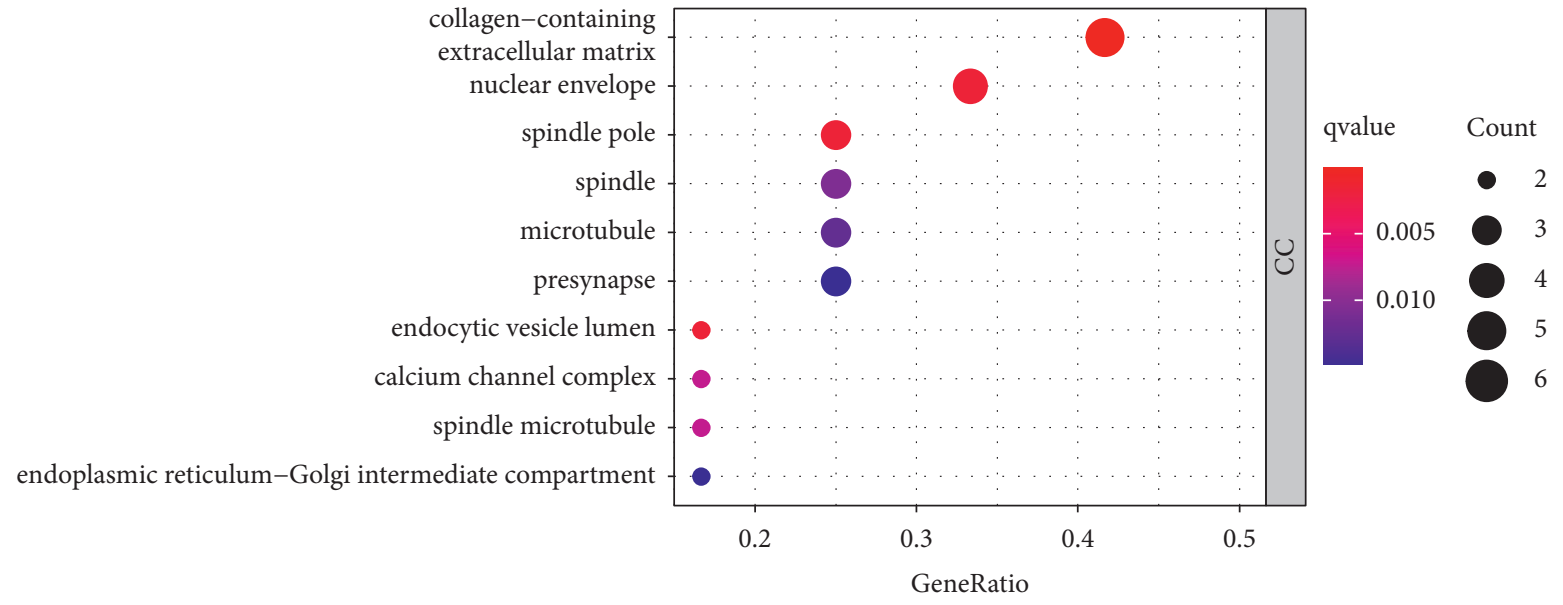

(b)

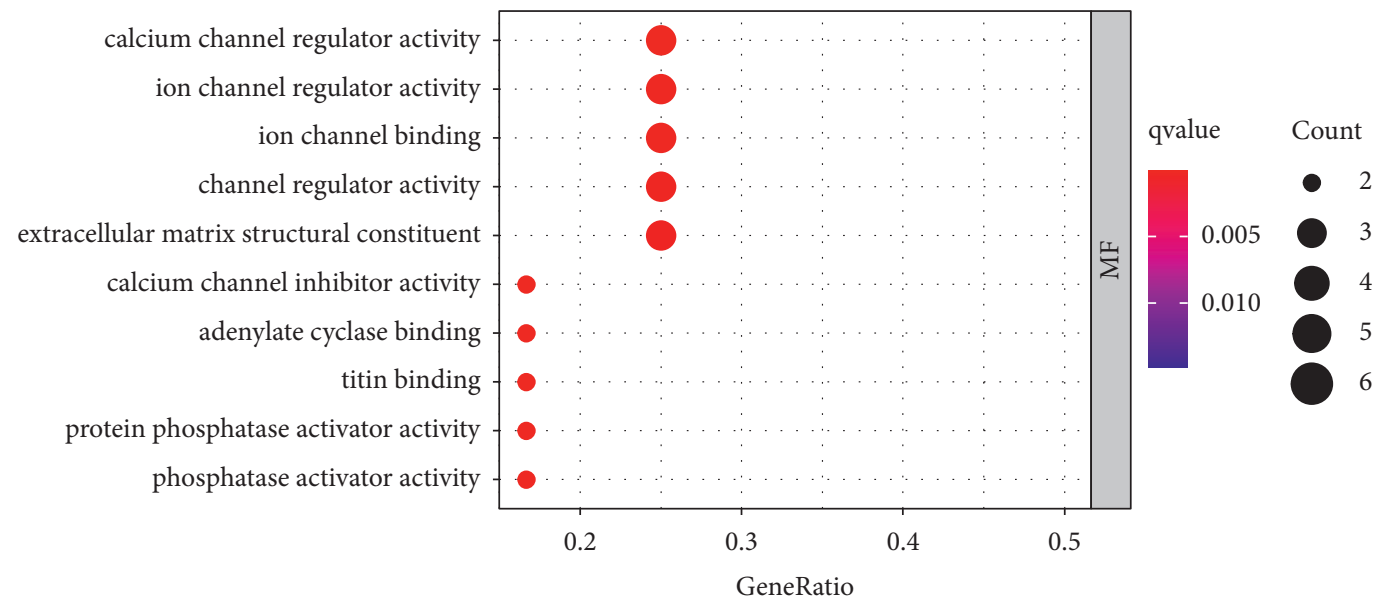

(c)

FIgURE 3: GO functional annotation of 12 intersected targets. (a) The 10 most significant BP terms. (b) The 10 most significant CC terms. (c) The 10 most significant MF terms. 


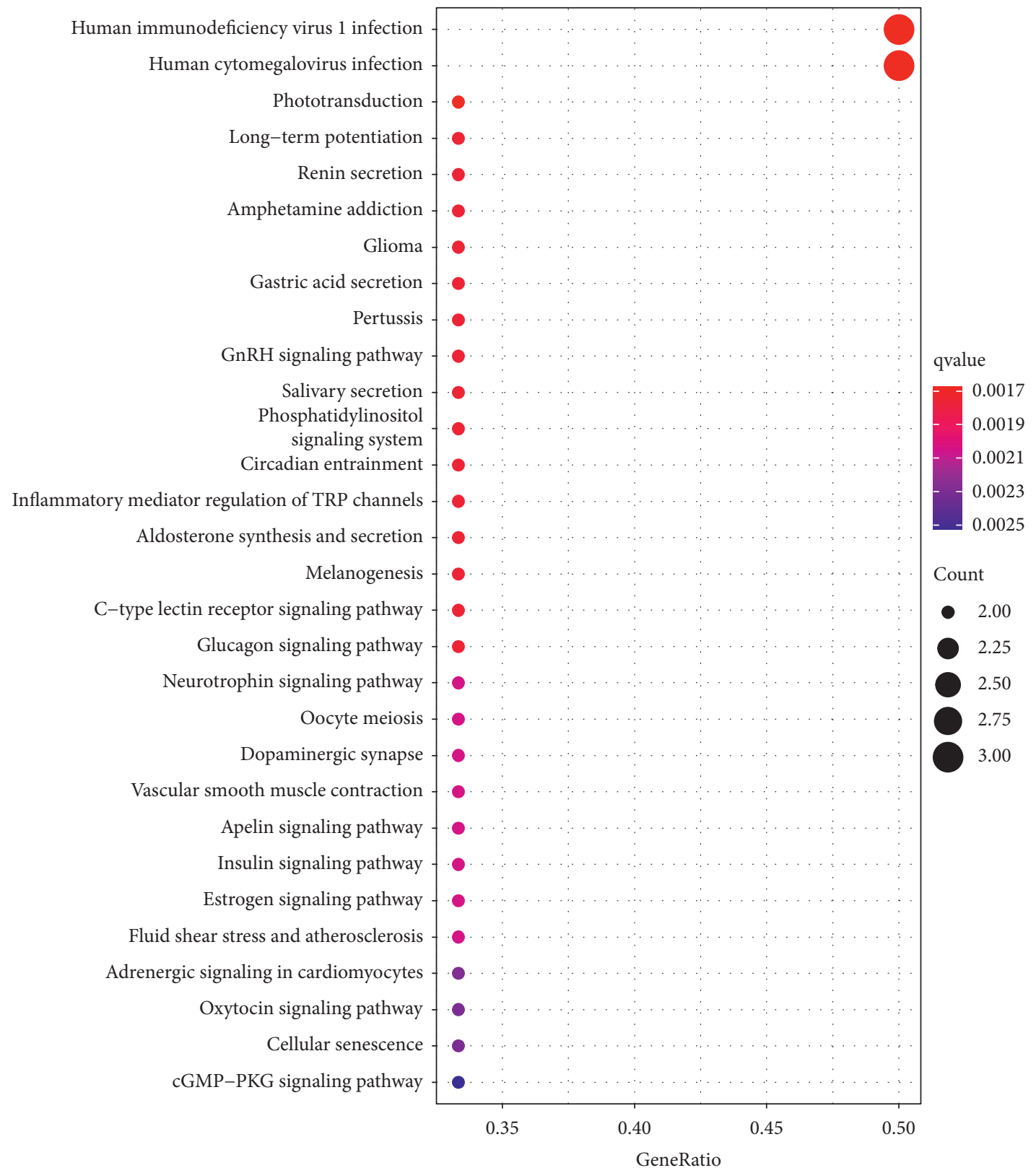

FIGURE 4: KEGG pathways for the 12 intersected targets.

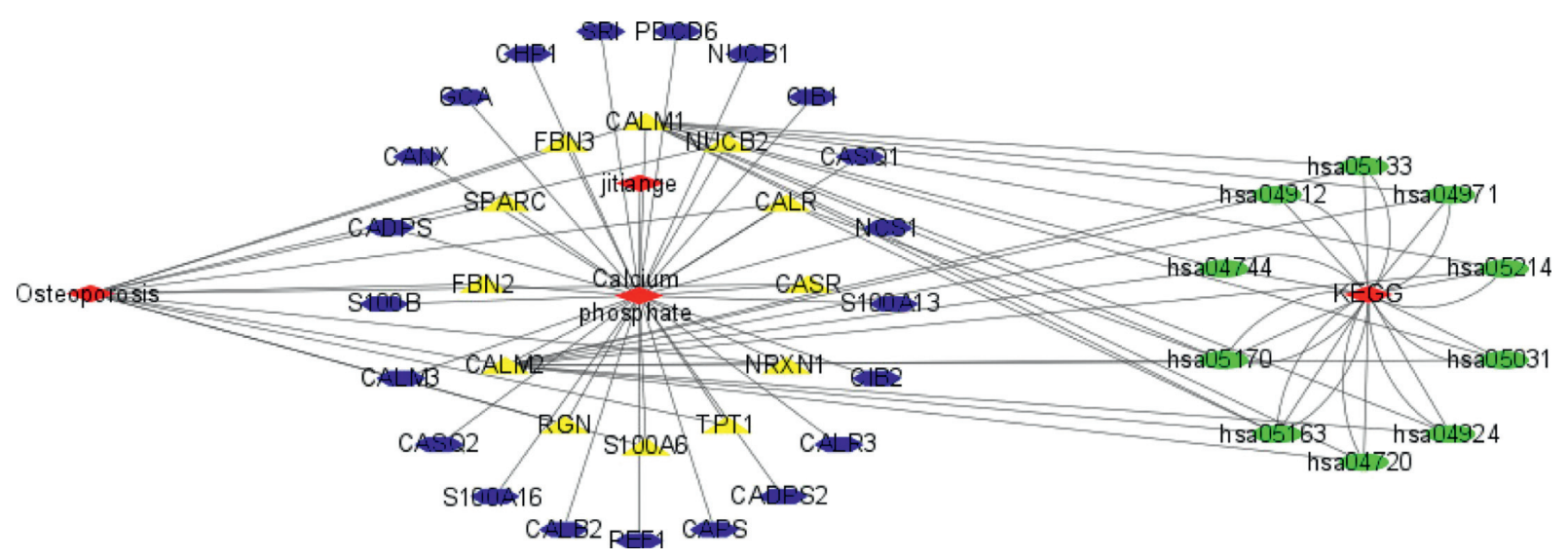

Figure 5: D-D-I-T-P networks. 

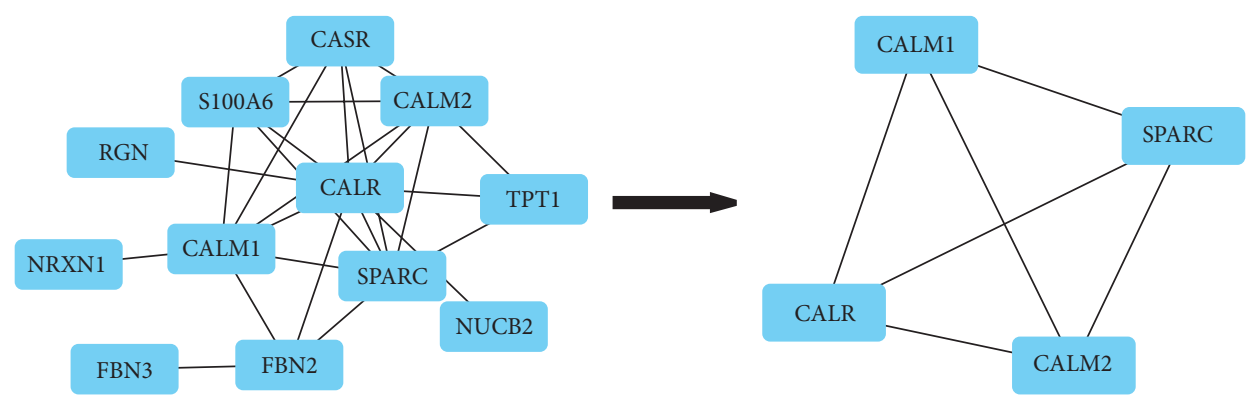

Figure 6: According to the analysis with CytoNCA, the core targets were obtained.

TABLE 2: Information of 4 core targets.

\begin{tabular}{lccc}
\hline Uniprot ID & Target genes & Protein & Degree \\
\hline P27797 & CALR & Calreticulin & 9 \\
P09486 & SPARC & SPARC & 7 \\
P0DP23 & CALM1 & Calmodulin-1 & 7 \\
P0DP24 & CALM2 & Calmodulin-2 & 6 \\
\hline
\end{tabular}

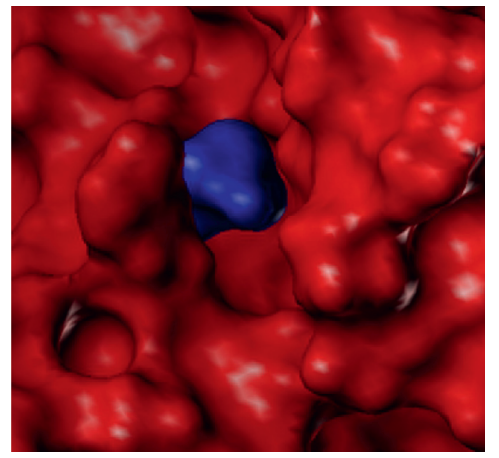

(a)

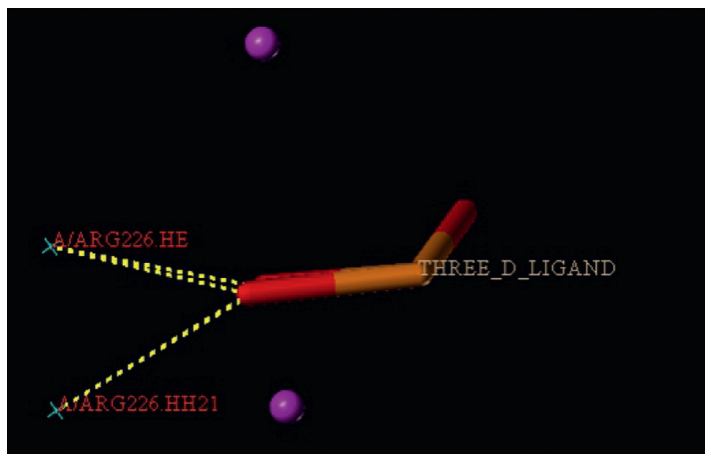

(b)

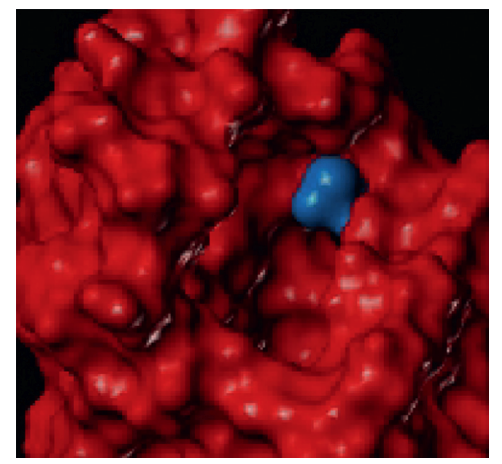

(c)

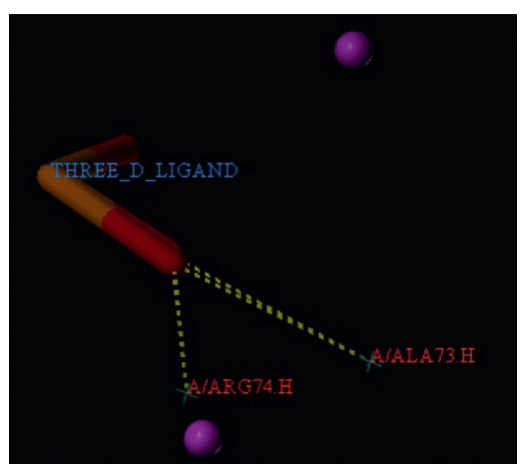

(d)

Figure 7: Calcium phosphate: $(\mathrm{a}, \mathrm{b})$ CALR protein; $(\mathrm{c}, \mathrm{d})$ CALM1 protein.

TABLE 3: Results of molecular docking of calcium phosphate with 2 proteins.

\begin{tabular}{lcccccc}
\hline \multirow{2}{*}{ Ingredient } & \multicolumn{3}{c}{ CALR } & \multicolumn{3}{c}{ CALM1 } \\
& Total score & Crash & Polar & Total score & Crash & Polar \\
\hline Calcium phosphate & 6.9181 & -0.3732 & 7.6852 & 5.3811 & -0.3267 & 6.9826 \\
\hline
\end{tabular}


containing extracellular matrix, nuclear envelope, spindle, and calcium channel complex. Simultaneously, MF mainly contained calcium channel regulator activity, ion channel binding, cytokine activity, and phosphatase activator activity.

In addition, we observed that 45 pathways were related to these overlapping targets. As revealed by the KEGG enrichment analysis, those identified key targets were mostly associated with Rap1, Ras, cAMP, cGMP-PKG, calcium signaling pathways, and so on. In recent studies, the cGMPPKG pathway is suggested to modulate the growth and differentiation of osteoblasts by forming the mechanosome that contains Src [15]. As a result, it may exert a critical role in skeletal homeostasis [16]. Rap1 contributes to activating the processes such as clustering of integrin, adhesion of cells onto the bone matrix, transembrane transduction, resultant signal transduction, and cytoskeletal modifications. Rap1 deficiency influences bone resorption in vivo, leading to OP occurrence [17]. The Rap1 pathway determines the osteogenesis or adipogenesis of BMSCs, which clarifies the bone metabolic alterations during OP progression [18]. The increased cAMP/cGMP expression within osteoblasts can induce osteogenesis [19]. RANKL and Cbfal contribute to the key factors that maintain bone homeostasis and regulate the differentiation of osteoclasts and osteoblasts, which can be achieved through the cAMP pathway. Angiotensin II and factors such as high/low density lipoprotein (HDL/LDL), homocysteine, and nitric oxide (NO) that are frequently changed in the case of OP and hypertension decreases the Cbfal level and increases the RANKL level through the cAMP pathway [20]. Ras pathway has a key function in maintaining the bone stability. After osteocytes are stimulated mechanically, factors related to this pathway are upregulated [21]. Moreover, the Ras pathway modulates immature bone progenitor cell growth and differentiation to osteoblasts, and the activated pathway contributes to activating the downstream PI3K-AKT and MAPK pathways for the enhancement of bone density [22]. The elevation of intracellular calcium levels could activate the calmodulin $(\mathrm{CaM}) /$ calmodulin-dependent protein kinase (CaMK) pathway and regulate osteoclast differentiation [23]. According to metabolic analysis and RNA sequencing, the activated Mincle promotes the genesis of osteoclasts by the ITAM-based calcium pathway, which skews the metabolism of osteoclasts to oxidative phosphorylation [24]. Arctiin may suppress the genesis of osteoclasts through inactivating the RANKL-triggered calcium pathway and upregulating the ROS-scavenging enzyme expression related to the Nrf2/ Keap1/ARE pathway, which can thus prevent bone loss caused by ovariectomy [25].

As an important research method, D-D-I-T-P networks could better demonstrate the relationship between disease, drug, ingredients, targets, and pathways. In our study, we constructed the current networks. According to the analysis with CytoNCA, we acquired 4 core targets including CALR, SPARC, CALM1, and CALM2.

As the calcium-binding chaperone, CALR targets the calreticulin/calnexin cycle to enhance endoplasmic reticulum (ER) folding, quality control, and oligomeric assembly.
Such lectin exhibits transient interaction with nearly every monoglucosylated glycoprotein produced within ER [26]. Additionally, it binds to the NR3C1 DNA-binding domain in order to regulate the transcriptional activity of steroid hormone receptors [27]. CALM1 is responsible for the mediation of L-type calcium channel depending on voltage [28], regulation of CACNA1C inactivation depending on calcium [29], positive regulation of KCNN2's potassium channel activity activated by calcium [30], formation of the KCNQ1-based potassium channel complex, and regulation of channel electrophysiological activities by binding to calcium [31]. Besides, it dominates a role of a sensor for modulating the contact between ER and additional organelles in a VMP1:ATP2A2-mediated manner [32]. Moreover, calmodulin binds to calcium to control numerous ion channels, enzymes, proteins, and aquaporins. Numerous phosphatases and protein kinases are triggered by the calmodulin-calcium complex. Centrin and CCP110 are associated with the genetic pathway regulating centrosome progression and cycle by means of cytokinesis [33]. By performing $\mathrm{MD}$ analysis, the main ingredient, calcium phosphate, had good binding with the CALR protein and CALM1 protein. The abovementioned results suggested that the NP-selected major ingredients are reliable and are related to the targets for OP.

\section{Conclusion}

To conclude, this study found that 4 main ingredients of the jintiange capsule, particularly calcium phosphate, could act on 33 targets. Totally, 4745 potential disease targets for OP and 12 overlapping targets were identified by the approach of NP. Then, we constructed a PPI network of these targets, conducted the enrichment analysis of GO and KEGG, constructed D-D-I-T-P networks, obtained 4 core targets, and performed MD experiments to investigate the potential mechanism of the jintiange capsule for treating OP. In summary, the main ingredient of jintiange capsule, particularly calcium phosphate, may interact with 2 targets, CALR and CALM1, and regulate multiple signaling pathways to treat OP. Although these findings based on NP have great significance for understanding the potential mechanism of the jintiange capsule for treating OP and discovering new effective ingredients, there remain some limitations in our study. The obtained findings are only theoretical predictions. In the future, a large number of experiments in vivo or in vitro are still needed to verify these findings.

\section{Data Availability}

The figures and tables used to support the findings of this study are included within the article, and the original data are available from the first author or corresponding author upon request.

\section{Disclosure}

Zhao Yang and Zhen-zhen Yuan are the co-first authors. 


\section{Conflicts of Interest}

The authors declare that there are no conflicts of interest regarding the publication of this paper.

\section{Authors' Contributions}

Zhao Yang and Zhen-Zhen Yuan contributed equally to this work.

\section{References}

[1] P. P. Coll, S. Phu, S. H. Hajjar, B. Kirk, G. Duque, and P. Taxel, "The prevention of osteoporosis and sarcopenia in older adults," Journal of the American Geriatrics Society, vol. 69, no. 5, pp. 1388-1398, 2021.

[2] J.-Y. Noh, Y. Yang, and H. Jung, "Molecular mechanisms and emerging therapeutics for osteoporosis," International Journal of Molecular Sciences, vol. 21, no. 20, p. 7623, 2020.

[3] Z. Farrah and A. S. Jawad, "Optimising the management of osteoporosis," Clinical Medicine, vol. 20, no. 5, pp. e196-e201, 2020.

[4] X. Wang, Z.-Y. Wang, J.-H. Zheng, and S. Li, “TCM network pharmacology: a new trend towards combining computational, experimental and clinical approaches," Chinese Journal of Natural Medicines, vol. 19, no. 1, pp. 1-11, 2021.

[5] J. Sun, X. G. Yang, and Y. C. Hu, "Efficacy of jintiange capsules in the treatment of osteoporosis: a network meta-analysis," Orthopaedic Surgery, vol. 11, no. 2, pp. 176-186, 2019.

[6] A. L. Hopkins, "Network pharmacology," Nature Biotechnology, vol. 25, no. 10, pp. 1110-1111, 2007.

[7] X. Zhang, T. Shen, X. Zhou et al., "Network pharmacology based virtual screening of active constituents of Prunella vulgaris L. and the molecular mechanism against breast cancer," Scientific Reports, vol. 10, no. 1, p. 15730, 2020.

[8] S. Saikia and M. Bordoloi, "Molecular docking: challenges, advances and its use in drug discovery perspective," Current Drug Targets, vol. 20, no. 5, pp. 501-521, 2019.

[9] Q. Tao, J. Du, X. Li et al., "Network pharmacology and molecular docking analysis on molecular targets and mechanisms of Huashi Baidu formula in the treatment of COVID-19," Drug Development and Industrial Pharmacy, vol. 46, no. 8, pp. 1345-1353, 2020.

[10] L. Huang, D. Xie, Y. Yu et al., "TCMID 2.0: a comprehensive resource for TCM," Nucleic Acids Research, vol. 46, no. D1, pp. D1117-D1120, 2018.

[11] T. Fang, L. Liu, and W. Liu, "Network pharmacology-based strategy for predicting therapy targets of Tripterygium wilfordii on acute myeloid leukemia," Medicine, vol. 99, no. 50, Article ID e23546, 2020.

[12] S. Christakos, P. Dhawan, A. Verstuyf, L. Verlinden, and G. Carmeliet, "Vitamin D: metabolism, molecular mechanism of action, and pleiotropic effects," Physiological Reviews, vol. 96, no. 1, pp. 365-408, 2016.

[13] M. Amling, M. Priemel, T. Holzmann et al., "Rescue of the skeletal phenotype of vitamin $\mathrm{D}$ receptor-ablated mice in the setting of normal mineral ion homeostasis: formal histomorphometric and biomechanical analyses 1," Endocrinology, vol. 140, no. 11, pp. 4982-4987, 1999.

[14] R. Rizzoli, "Vitamin D supplementation: upper limit for safety revisited?" Aging Clinical and Experimental Research, vol. 33, no. 1, pp. 19-24, 2021.

[15] W. Wisanwattana, K. Wongkrajang, D.-Y. Cao et al., "Inhibition of phosphodiesterase 5 promotes the aromatase- mediated estrogen biosynthesis in osteoblastic cells by activation of cGMP/PKG/SHP2 pathway," Frontiers in Endocrinology, vol. 12, Article ID 636784, 2021.

[16] S. M. Kim, T. Yuen, J. Iqbal, M. R. Rubin, and M. Zaidi, “The NO-cGMP-PKG pathway in skeletal remodeling," Annals of the New York Academy of Sciences, vol. 1487, no. 1, pp. 21-30, 2021.

[17] K. Jeevaratnam, S. C. Salvage, M. Li, and C. L.-H. Huang, "Regulatory actions of $3^{\prime}, 5^{\prime}$-cyclic adenosine monophosphate on osteoclast function: possible roles of Epac-mediated signaling," Annals of the New York Academy of Sciences, vol. 1433, no. 1, pp. 18-28, 2018.

[18] J. Wu, P. Cai, Z. Lu et al., "Identification of potential specific biomarkers and key signaling pathways between osteogenic and adipogenic differentiation of hBMSCs for osteoporosis therapy," Journal of Orthopaedic Surgery and Research, vol. 15, no. 1, p. 437, 2020.

[19] K. Porwal, S. Pal, S. Bhagwati, M. I. Siddiqi, and N. Chattopadhyay, "Therapeutic potential of phosphodiesterase inhibitors in the treatment of osteoporosis: scopes for therapeutic repurposing and discovery of new oral osteoanabolic drugs," European Journal of Pharmacology, vol. 899, Article ID 174015, 2021.

[20] X.-X. Guan, Y. Zhou, and J.-Y. Li, "Reciprocal roles of angiotensin II and Angiotensin II Receptors Blockade (ARB) in regulating Cbfa1/RANKL via cAMP signaling pathway: possible mechanism for hypertension-related osteoporosis and antagonistic effect of ARB on hypertension-related osteoporosis," International Journal of Molecular Sciences, vol. 12, no. 7, pp. 4206-4213, 2011.

[21] Y. Gebru, T.-Y. Diao, H. Pan, E. Mukwaya, and Y. Zhang, "Potential of RAS inhibition to improve metabolic bone disorders," BioMed Research International, vol. 2013, Article ID 932691, 6 pages, 2013.

[22] G. Papaioannou, F. Mirzamohammadi, and T. Kobayashi, "Ras signaling regulates osteoprogenitor cell proliferation and bone formation," Cell Death \& Disease, vol. 7, no. 10, p. e2405, 2016.

[23] W. Liu, C. C. Le, D. Wang et al., "Ca2+/CaM/CaMK signaling is involved in cadmium-induced osteoclast differentiation," Toxicology, vol. 441, Article ID 152520, 2020.

[24] D. Andreev, M. Liu, D. Weidner et al., "Osteocyte necrosis triggers osteoclast-mediated bone loss through macrophageinducible C-type lectin," Journal of Clinical Investigation, vol. 130, no. 9, pp. 4811-4830, 2020.

[25] D. Chen, Z. Ye, C. Wang et al., "Arctiin abrogates osteoclastogenesis and bone resorption via suppressing RANKL-induced ROS and NFATc1 activation," Pharmacological Research, vol. 159, Article ID 104944, 2020.

[26] W. M. Nauseef, S. J. McCormick, and R. A. Clark, "Calreticulin functions as a molecular chaperone in the biosynthesis of myeloperoxidase," Journal of Biological Chemistry, vol. 270, no. 9, pp. 4741-4747, 1995.

[27] J. M. Holaska, B. E. Black, D. C. Love, J. A. Hanover, J. Leszyk, and B. M. Paschal, "Calreticulin Is a receptor for nuclear export," Journal of Cell Biology, vol. 152, no. 1, pp. 127-140, 2001.

[28] L. M. Wren, J. Jiménez-Jáimez, S. Al-Ghamdi et al., "Genetic mosaicism in calmodulinopathy," Circulation: Genomic and Precision Medicine, vol. 12, no. 9, pp. 375-385, 2019.

[29] N. J. Boczek, N. Gomez-Hurtado, D. Ye et al., "Spectrum and prevalence of CALM1 -, CALM2 -, and CALM3 -encoded calmodulin variants in long QT syndrome and functional characterization of a novel long QT syndrome-associated 
calmodulin missense variant, E141G," Circulation: Cardiovascular Genetics, vol. 9, no. 2, pp. 136-146, 2016.

[30] C.-C. Yu, J.-S. Ko, T. Ai et al., “Arrhythmogenic calmodulin mutations impede activation of small-conductance calciumactivated potassium current," Heart Rhythm, vol. 13, no. 8, pp. 1716-1723, 2016.

[31] D. Sachyani, M. Dvir, R. Strulovich et al., "Structural basis of a Kv7.1 potassium channel gating module: studies of the intracellular c-terminal domain in complex with calmodulin," Structure, vol. 22, no. 11, pp. 1582-1594, 2014.

[32] Y. G. Zhao, Y. Chen, G. Miao et al., "The ER-localized transmembrane protein EPG-3/VMP1 regulates SERCA activity to control ER-isolation membrane contacts for autophagosome formation," Molecular Cell, vol. 67, no. 6, pp. 974-989, 2017.

[33] W. Y. Tsang, A. Spektor, D. J. Luciano et al., "CP110 cooperates with two calcium-binding proteins to regulate cytokinesis and genome stability," Molecular Biology of the Cell, vol. 17, no. 8, pp. 3423-3434, 2006. 